\title{
The Role of Needle-Based Confocal Laser Endomicroscopy in the Evaluation of Pancreatic Cystic Lesions: A Systematic Review
}

\author{
Yung Ka Chin ${ }^{1}$, Clement Chun Ho Wu ${ }^{1,2}$ and Damien Meng Yew Tan ${ }^{1,2}$ \\ ${ }^{1}$ Department of Gastroenterology and Hepatology, Singapore General Hospital, Singapore, ${ }^{2}$ Duke-NUS Medical School, Singapore, \\ Singapore
}

The prevalence of pancreatic cystic lesions (PCLs) has increased recently due to the increased use of cross-sectional abdominal imaging and the ageing global population. Current diagnostic techniques are inadequate to distinguish between PCLs that require surgery, close surveillance, or expectant management. This has resulted in increased morbidity from both inappropriately aggressive and conservative management strategies. Needle-based confocal laser endomicroscopy (nCLE) has allowed microscopic examination and visual delineation of the surface epithelium of PCLs. Landmark studies in this decade have correlated nCLE and histological findings and identified characteristics differentiating various types of PCLs. Subsequent studies have confirmed the high diagnostic yield of nCLE and its diagnostic utility in PCLs with an equivocal diagnosis. Moreover, nCLE has been shown to improve the diagnostic yield of PCLs. This will help avoid unnecessary pancreatic surgery, which carries significant morbidity and mortality risks. The early detection of high-grade dysplasia in PCLs will provide early surgical treatment and improve outcomes for pancreatic cancer. Despite the high upfront cost of nCLE, the improved diagnostic accuracy and resultant appropriate management have resulted in improved cost effectiveness. Refining the procedure technique and limiting the procedure length have significantly improved the safety of nCLE. A structured training program and device improvements to allow more complete mapping of the pancreatic cyst epithelium will be crucial for the widespread adoption of this promising technology. Clin Endosc 2021;54:38-47

Key Words: Cost effectiveness; Endoscopic ultrasound; Fine needle aspiration; Needle-based confocal laser endomicroscopy; Pancreatic cyst

\section{INTRODUCTION}

Cystic lesions of the pancreas are increasingly detected because of the increased use of cross-sectional abdominal imaging such as computed tomography and magnetic resonance imaging. ${ }^{1}$ Pancreatic cysts are mostly incidentally identified when abdominal imaging is performed for unrelated indications. ${ }^{2}$ The prevalence of incidental pancreatic cysts is $2.6 \%$,

\footnotetext{
Received: October 19, 2019 Accepted: January 6, 2020

Correspondence: Damien Meng Yew Tan

Department of Gastroenterology and Hepatology, Singapore General Hospital, Outram Road, Singapore 169608, Singapore

Tel: +65-9646-2325, Fax: +65-6227-3623, E-mail: Damien.tan.m.y@singhealth. com.sg

ORCID: https://orcid.org/0000-0002-7282-0900

It is the invited review article.

(c) This is an Open Access article distributed under the terms of the Creative Commons Attribution Non-Commercial License (http://creativecommons.org/ licenses/by-nc/3.0) which permits unrestricted non-commercial use, distribution, and reproduction in any medium, provided the original work is properly cited.
}

with an increased prevalence in the elderly of approximately $8 \%$ as described by de Jong et al. ${ }^{3}$ Little information is presently available on the natural history of pancreatic cysts, which can be classified as benign, pre-malignant, or malignant. However, making an accurate discrimination among these categories using the current diagnostic techniques remains challenging. ${ }^{4}$

Prior to the introduction of clinical guidelines by the International Association of Pancreatology $y^{5}$ and American College of Gastroenterology ${ }^{6}$ differentiating the management of various subtypes of pancreatic cysts, the surgical resection rates of pancreatic cysts were high. A study by Goh et al. showed that prior to 2006, approximately $40 \%$ of patients who underwent surgery for asymptomatic pancreatic cystic lesions (PCLs) had benign cysts. ${ }^{7}$ This meant that a significant proportion of patients were undergoing major surgery, which involved a $5 \%$ risk of mortality and up to $60 \%$ risk of morbidity ${ }^{8}$ and incurred unnecessary medical costs, detrimentally affecting quality of life. ${ }^{9}$ 
Updated clinical practice guidelines by major societies have sought to optimize the diagnosis and management of PCLs. ${ }^{10-}$ ${ }^{12}$ Cross-sectional imaging is useful for identifying worrisome features and high-risk stigmata, but major society guidelines have recommended further delineation via endoscopic ultrasound-fine needle aspiration (EUS-FNA) to accurately ascertain the nature of lesions with worrisome features. ${ }^{10-12}$ EUS accurately determines the location of the cyst within the pancreas (head vs. body vs. tail) and the relationship with the pancreatic duct and cyst structure (unilocular vs. macrocystic vs. microcystic); however, these features alone are not diagnostic. FNA enables the collection of cyst fluid for cytological examination and tumor markers such as carcinoembryonic antigen (CEA) for further characterization, but this is limited by the often acellular nature of cyst fluid and the high sensitivity albeit low specificity of CEA in the diagnosis of mucinous cysts. ${ }^{13,14}$ The combination of the three main diagnostic modalities (cross-sectional imaging, fluid cytology, and fluid CEA) was reported by Brugge et al. to have an accuracy of only $70 \%{ }^{15}$ Hence, there is a pressing need for a better diagnostic method to improve the diagnostic accuracy of PCLs.

Confocal laser endomicroscopy (CLE) involves the use of a low-power laser to scan and illuminate tissue within a pancreatic cyst in a single focal plane, allowing for microscopic detail of the surface epithelium to be examined. Light is focused and passed through a confocal aperture. This reduces light scatter above and below the plane. The microscopic details of the surface epithelium can be examined, ${ }^{16}$ and characteristics distinguishing various pancreatic cyst types can be appreciated.

Due to its extraluminal location, the pancreas is difficult to assess using the probe-based CLE method; therefore, a needle-based CLE (nCLE) was designed to overcome these technical difficulties assessing cystic lesions of the pancreas. ${ }^{17}$ In fact, nCLE enables real-time histopathology assessments of cystic pancreatic lesions. This may reduce sampling error and can consequently reduce the number of passes needed for diagnosis.

\section{METHOD}

Two authors individually reviewed the English language literature from inception to November 2019. PubMed and Google Scholar were used to identify peer-reviewed original articles using the following keywords: "needle-based confocal laser endomicroscopy", "endoscopic ultrasound", and "pancreatic cystic lesion". Among the identified results, we included original research papers and cases series (consisting of at least three cases) that reported the use of nCLE. We also manually searched the references lists of the pertinent studies to identify additional relevant studies. Only studies involving humans were selected. We excluded non-English papers, reviews, and papers that were unrelated to the issue at hand or were published only as abstracts. Retrieved duplicates were excluded. Indications, procedural details, technical and clinical outcomes, and adverse events and their management were reviewed for each study.

\section{Device and procedure}

\section{nCLE probe}

The AQ-Flex 19 miniprobe model (Fig. 1) (Cellvizio, Mauna Kea Technologies, Paris, France) has 10,000 optical fibers enabling a field of view of $320 \mu \mathrm{m}$ and a lateral resolution of $3.5 \mu \mathrm{m}$. The probe measures a $0.85-\mathrm{mm}$ diameter and is $4 \mathrm{~m}$ long. It is compatible with 19-gauge EUS-FNA needles. Imaging depth is the focal plane, which is scanned by confocal microscopy system. The probes have fixed optical properties in the distal tip that focus illumination and detection on a single focal plane.

Three safety features have been integrated into this miniprobe (Fig. 2). First, the ferule, which is the metallic tip at the distal end of the probe, protects the device from the beveled tip of the needle and ensures device integrity. The ferule is 4 $\mathrm{mm}$ long and ensures that the microprobe can be safely inserted into and extracted from the needle. Second, the sheath of the probe comprises a robust protective biocompatible coating. Finally, the probe's position within the FNA needle is maintained by a locking device that is attached by a Luer lock to the FNA needle's proximal hub and secured to the probe. This prevents the tip of the probe from migrating farther than intended from the needle.

\section{Procedure}

Before the beginning of the procedure, the locking device is attached to the proximal end of a 19-G FNA needle after removal of the stylet. A list of FNA needles compatible with the nCLE miniprobe (AQ-Flex; Cellvizio, Mauna Kea Technologies) is shown in Table 1. Antibiotic prophylaxis is administered prior to commencement of the procedure. The miniprobe is introduced into the needle via the locking device. The miniprobe is then advanced through the needle until its tip protrudes beyond the needle's bevel. The miniprobe is then retracted slightly into the needle and the needle and miniprobe are inserted together into the endoscope. After the pancreatic cyst is identified and punctured by the needle, the miniprobe is advanced and brought into contact with the cyst wall or solid component (if present). The intravenous injection of 2.5 $\mathrm{mL}$ of fluorescein $10 \%$ is administered to enable fluorescent imaging. Microscopic real-time sequences are then recorded 


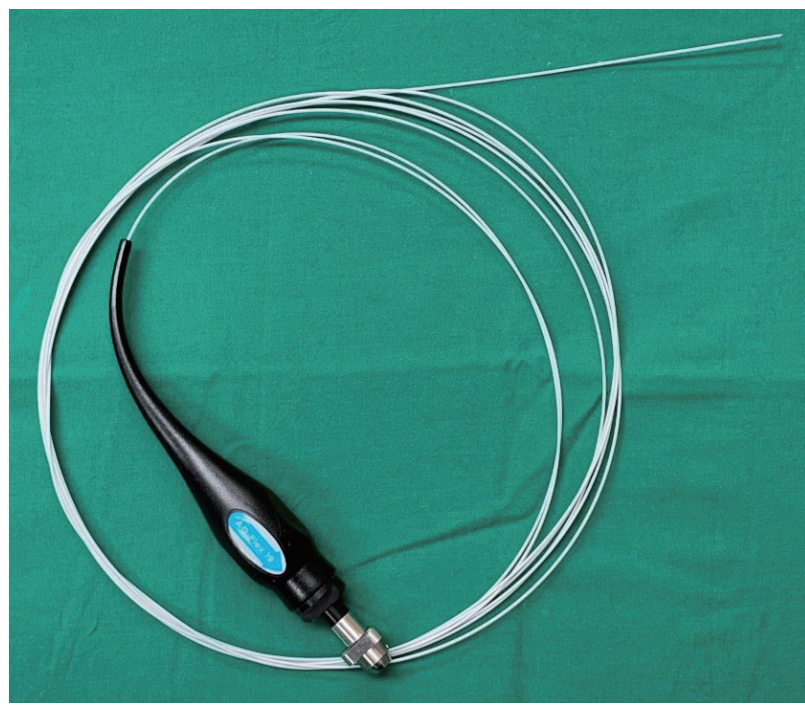

Fig. 1. The AQ-Flex (Cellvizio, Mauna Kea Technologies, Paris, France) 19 needle confocal laser endomicroscopy miniprobe.

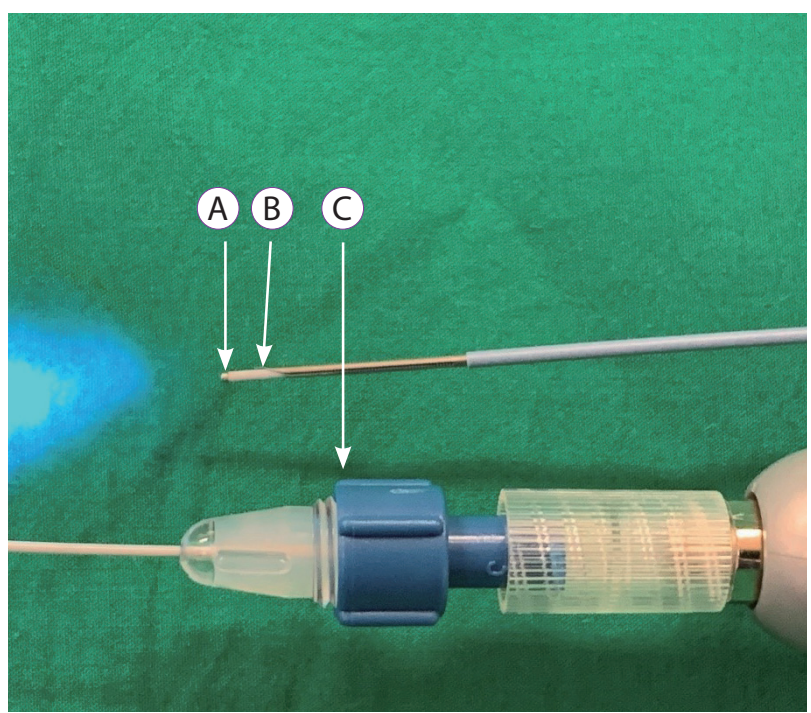

Fig. 2. (A) The ferule is a metallic tap at the distal end of the probe that protects the device from the beveled needle tip. (B) The sheath of the probe comprises a robust protective biocompatible coating. (C) A locking device is attached by a Luer lock to the fine needle aspiration needle's proximal hub and secured onto the probe to maintain needle position and prevent migration.

Table 1. Compatibility of Endoscopic Ultrasound-Fine Needle Aspiration Needle with the Needle-Based Confocal Laser Endomicroscopy Probe

\begin{tabular}{|c|c|c|}
\hline Needle type & Manufacturer & Compatibility \\
\hline EchoTip ${ }^{\circledR}$ Ultra Endoscopic Ultrasound Needle & Cook Medical & Compatible \\
\hline EZShot 2 Aspiration Needle & Olympus & Compatible \\
\hline Expect ${ }^{\mathrm{TM}}$ Needle 19 Flex & Boston Scientific & Compatible \\
\hline SonoTip ${ }^{\circledR}$ Pro Control & MediGlobe Gmbh & Compatible \\
\hline SonoTip ${ }^{\circledR}$ II & MediGlobe Gmbh & Compatible \\
\hline BNX Fine Needle Aspiration System & Beacon & Compatible \\
\hline EchoTip ${ }^{\circledR}$ Ultra Endoscopic Ultrasound Access Needle & Cook Medical & Not compatible \\
\hline EchoTip ${ }^{\circledR}$ ProCore & Cook Medical & Not compatible \\
\hline Quick-Core ${ }^{\circledR}$ Endoscopic Ultrasound Needle & Cook Medical & Not compatible \\
\hline 19 G FlexNeedle ${ }^{\mathrm{TM}}$ Clearview & CONMED & Not compatible \\
\hline
\end{tabular}

All needles are 19 G. Adapted from Mauna Kea Technologies, Paris, France.

for up to 10 minutes while the needle is within the pancreatic cyst, preferably against the cyst wall, to image the cellular structures. The microprobe is extracted from the needle after a diagnosis is made using nCLE imaging. Cyst fluid is then aspirated and sent for biochemical, cytological, and/or tumor marker analysis.

\section{REVIEW OF THE EVIDENCE}

The landmark clinical studies examining the role of nCLE in the diagnosis of pancreatic cysts are summarized in Table 2. The diagnostic features of the main subtypes of PCLs and their validity scores (sensitivity, specificity, and accuracy) are summarized in Table 3. The clinical application of nCLE in PCLs was first reported by Konda et al. in 2011 as a feasibility study. ${ }^{17}$ The post-insertion loading technique and long ferule 
Table 2. A Summary of the Key Clinical Studies Examining the Use of Needle-Based Confocal Laser Endomicroscopy for Diagnosing Pancreatic Cystic Lesions

\begin{tabular}{|c|c|c|c|c|c|c|c|c|}
\hline Study & Study design & Country & $\begin{array}{l}\text { EUS-nCLE } \\
\text { accuracy }\end{array}$ & Basis of final diagnosis & $n$ & $\begin{array}{l}\text { Sex } \\
(M / F)\end{array}$ & $\begin{array}{l}\text { Age } \\
\text { (mean in } \\
\text { years) }\end{array}$ & $\begin{array}{l}\text { Size } \\
(\mathrm{mm})\end{array}$ \\
\hline $\begin{array}{l}\text { Konda et al. }(2011)^{17} \\
\text { (INSPECT) }\end{array}$ & Prospective & USA & $\begin{array}{l}\text { Safety and } \\
\text { feasibility } \\
\text { study }\end{array}$ & $\begin{array}{l}\text { - Surgical histology } \\
\text { - EUS-FNA cytology } \\
\text { - EUS imaging }\end{array}$ & 18 & $7 / 11$ & 57.9 & 43 \\
\hline $\begin{array}{l}\text { Nakai et al. }(2015)^{19} \\
\text { (DETECT) }\end{array}$ & Prospective & USA & $89 \%$ & $\begin{array}{l}\text { - Surgical histology } \\
\text { - Cross-sectional imaging } \\
\text { - EUS imaging } \\
\text { - Cyst fluid analysis } \\
\text { - EUS-FNA cytology }\end{array}$ & 30 & $9 / 21$ & 72 & 30 \\
\hline $\begin{array}{l}\text { Napoléon et al. }(2015)^{20} \\
\text { (CONTACT 1) }\end{array}$ & Prospective & France & $87 \%$ & $\begin{array}{l}\text { - Surgical histology } \\
\text { - EUS-FNA cytology } \\
\text { - Multi-disciplinary consensus }\end{array}$ & 31 & $6 / 25$ & 57 & 39 \\
\hline Karia et al. $(2016)^{25}$ & Retrospective & USA & $46 \%$ & $\begin{array}{l}\text { - Surgical histology } \\
\text { - Cross-sectional imaging } \\
\text { - EUS imaging } \\
\text { - Cyst fluid analysis } \\
\text { - EUS-FNA cytology }\end{array}$ & 15 & $10 / 5$ & 66.6 & 25 \\
\hline Krishna et al. $(2016)^{27}$ & Retrospective & USA & $95 \%$ & $\begin{array}{l}\text { - Surgical histology } \\
\text { - EUS-FNA cytology } \\
\text { - Multi-disciplinary consensus }\end{array}$ & 26 & $10 / 16$ & 54.8 & 32 \\
\hline Kadayifci et al. $(2017)^{26}$ & Retrospective & USA & $83 \%$ & $\begin{array}{l}\text { - Surgical histology } \\
\text { - EUS imaging } \\
\text { - Cyst fluid analysis }\end{array}$ & 18 & $8 / 10$ & 65.4 & 34 \\
\hline Krishna et al. $(2017)^{28}$ & Retrospective & USA & $95 \%$ & $\begin{array}{l}\text { - Surgical histology } \\
\text { - Multi-disciplinary consensus }\end{array}$ & 29 & $13 / 16$ & 53 & 32 \\
\hline $\begin{array}{l}\text { Napoleon et al. }(2019)^{23} \\
\text { (CONTACT 2) }\end{array}$ & Prospective & France & $91 \%$ & $\begin{array}{l}\text { - Surgical histology } \\
\text { - EUS imaging } \\
\text { - Cross-sectional imaging } \\
\text { - Cyst fluid analysis } \\
\text { - EUS-FNA cytology }\end{array}$ & 78 & $26 / 52$ & 57 & 40 \\
\hline Chin et al. $(2018)^{22}$ & Prospective & Singapore & $80 \%$ & $\begin{array}{l}\text { - Surgical histology } \\
\text { - EUS imaging } \\
\text { - Cross-sectional imaging } \\
\text { - Cyst fluid analysis } \\
\text { - EUS-FNA cytology }\end{array}$ & 12 & $6 / 6$ & 66.5 & 34 \\
\hline $\begin{array}{l}\text { Keane et al. }(2019)^{24} \\
(\text { CONCYST-01) }\end{array}$ & Prospective & $\begin{array}{l}\text { United } \\
\text { Kingdom }\end{array}$ & $77 \%$ & $\begin{array}{l}\text { - EUS imaging } \\
\text { - Cross-sectional imaging } \\
\text { - EUS-FNA cytology } \\
\text { - Multi-disciplinary consensus }\end{array}$ & 56 & $35 / 21$ & 68 & 25 \\
\hline Palazzo et al. $(2019)^{30}$ & Retrospective & France & $85 \%$ & $\begin{array}{l}\text { - Clinical factors } \\
\text { - Cross sectional imaging } \\
\text { - EUS imaging } \\
\text { - Cyst fluid analysis }\end{array}$ & 206 & $69 / 137$ & 57 & 38 \\
\hline
\end{tabular}

EUS-FNA, endoscopic ultrasound-fine needle aspiration; nCLE, needle-based confocal laser endomicroscopy. 
Table 3. A Summary of Needle-Based Confocal Laser Endomicroscopy Diagnostic Features for each Cystic Pathology as well as Their Sensitivity, Specificity, and Accuracy in Key Clinical Studies

\begin{tabular}{|c|c|c|c|c|c|}
\hline \multicolumn{6}{|l|}{ Serous cystadenoma } \\
\hline Pattern & Study & $n$ & Sensitivity & Specificity & Accuracy \\
\hline \multirow[t]{2}{*}{ Fern pattern } & Krishna et al. $(2017)^{28}$ & 13 & $98 \%$ & $97 \%$ & $92 \%$ \\
\hline & Krishna et al. $(2020)^{29}$ & 113 & $87 \%$ & $100 \%$ & $97 \%$ \\
\hline \multirow[t]{3}{*}{ Superficial vascular network } & Napoléon et al. $(2015)^{20}$ & 31 & $69 \%$ & $100 \%$ & $87 \%$ \\
\hline & Napoleon et al. $(2019)^{23}$ & 71 & $95 \%$ & $100 \%$ & $99 \%$ \\
\hline & Keane et al. $(2019)^{24}$ & 56 & $56 \%$ & - & $38 \%$ \\
\hline \multicolumn{6}{|l|}{ Pseudocyst } \\
\hline Pattern & Study & $n$ & Sensitivity & Specificity & Accuracy \\
\hline \multirow[t]{2}{*}{ Bright particles against dark background } & Napoleon et al. $(2016)^{21}$ & 31 & $43 \%$ & $100 \%$ & $87 \%$ \\
\hline & Krishna et al. $(2020)^{29}$ & 65 & $67 \%$ & $97 \%$ & $95 \%$ \\
\hline Bright grey and black particles & Keane et al. $(2019)^{24}$ & 56 & $67 \%$ & - & $67 \%$ \\
\hline \multicolumn{6}{|l|}{ Intraductal papillary mucinous neoplasm } \\
\hline Pattern & Study & $n$ & Sensitivity & Specificity & Accuracy \\
\hline \multirow[t]{4}{*}{ Finger-like papilla } & Nakai et al. $(2015)^{19}$ & 30 & $77 \%$ & $100 \%$ & $87 \%$ \\
\hline & Napoleon et al. $(2016)^{21}$ & 31 & $80 \%$ & $92 \%$ & $90 \%$ \\
\hline & Krishna et al. $(2020)^{29}$ & 65 & $98 \%$ & $94 \%$ & $97 \%$ \\
\hline & Keane et al. $(2019)^{24}$ & 56 & $90 \%$ & - & $77 \%$ \\
\hline \multicolumn{6}{|l|}{ Mucinous cystic neoplasm } \\
\hline Pattern & Study & $n$ & Sensitivity & Specificity & Accuracy \\
\hline \multirow[t]{2}{*}{ Epithelial bands } & Napoleon et al. $(2016)^{21}$ & 31 & $67 \%$ & $96 \%$ & $90 \%$ \\
\hline & Krishna et al. $(2020)^{29}$ & 65 & $98 \%$ & $94 \%$ & $97 \%$ \\
\hline \multicolumn{6}{|c|}{ Mucinous cyst (both intraductal papillary mucinous neoplasm and mucinous cystic neoplasm) } \\
\hline Pattern & Study & $n$ & Sensitivity & Specificity & Accuracy \\
\hline \multirow[t]{3}{*}{ Finger-like papilla or epithelial bands } & Krishna et al. $(2015)^{33}$ & 33 & $91 \%$ & $95 \%$ & $94 \%$ \\
\hline & Krishna et al. $(2016)^{27}$ & 26 & $94 \%$ & $82 \%$ & $89 \%$ \\
\hline & Napoleon et al. $(2019)^{23}$ & 71 & $95 \%$ & $100 \%$ & $97 \%$ \\
\hline $\begin{array}{l}\text { Finger-like papilla or dark ring or gland-like structure } \\
\text { or epithelial band }\end{array}$ & Kadayifci et al. $(2017)^{26}$ & 18 & $66 \%$ & $100 \%$ & $83 \%$ \\
\hline $\begin{array}{l}\text { Rope ladder or branch vascular pattern or finger-like } \\
\text { papilla or epithelial bands }\end{array}$ & Krishna et al. $(2017)^{28}$ & 16 & $93 \%$ & $89 \%$ & $91 \%$ \\
\hline \multicolumn{6}{|l|}{ Neuroendocrine neoplasm } \\
\hline Pattern & Study & $n$ & Sensitivity & Specificity & Accuracy \\
\hline \multirow[t]{3}{*}{ Trabeculae of compact cells surrounded by grey tissue } & Krishna et al. $(2017)^{28}$ & 13 & $98 \%$ & $97 \%$ & $92 \%$ \\
\hline & Krishna et al. $(2020)^{29}$ & 65 & $100 \%$ & $96 \%$ & $96 \%$ \\
\hline & Napoleon et al. $(2019)^{23}$ & 71 & $100 \%$ & $95 \%$ & $96 \%$ \\
\hline \multicolumn{6}{|l|}{ Pancreatic cystic neoplasm } \\
\hline Pattern & Study & $n$ & Sensitivity & Specificity & Accuracy \\
\hline $\begin{array}{l}\text { Epithelial villous structures } \\
\text { - Finger-like papillary projections } \\
\text { - Dark ring with white core } \\
\text { - Gland-like or crypt-like structures }\end{array}$ & Konda et al. $(2013)^{18}$ & 66 & $59 \%$ & $100 \%$ & $71 \%$ \\
\hline
\end{tabular}


length were postulated to predispose patients to a higher rate of complications. After achieving technical feasibility and safety, a larger prospective pilot study (in vivo nCLE Study in the Pancreas with Endosonography of Cystic Tumors) of 66 patients who underwent nCLE imaging. ${ }^{18}$ In the first part of the study, nCLE images of pancreatic cysts from 26 patients were reviewed by an expert consensus panel. Features distinguishing various types of cysts were described and correlated to histological findings. The second part of the study assessed the performance of this nCLE expert consensus criteria in identifying pancreatic cystic neoplasms (PCNs) (including mucinous cystadenoma [MCA], intraductal papillary mucinous neoplasms [IPMNs]) or adenocarcinoma in 31 additional patients. The identification of epithelial villous structures via nCLE was associated with PCNs $(p=0.004)$ and had a sensitivity of $59 \%$, specificity of $100 \%$, positive predictive value of $100 \%$, and negative predictive value of $50 \%$. This suggested that nCLE has a high specificity for PCN detection but may be limited by its low sensitivity. Two cases (3\%) of pancreatitis were reported (95\% confidence interval [CI], 0.4\%-10.5\%).

In the Diagnosis of Pancreatic Cysts: Endoscopic Ultrasound, Through-the-Needle Confocal Laser Endomicroscopy and Cystoscopy Trial of Nakai et al., ${ }^{19}$ cystoscopy was performed using a through-the-needle fiber optic probe. This was combined with nCLE under EUS guidance to diagnose PCN. The sensitivities of cystoscopy and nCLE were $90 \%$ and $80 \%$, respectively. The combination of both methods yielded a sensitivity of $100 \%$ in 18 patients. This study had a significant post-procedure pancreatitis rate of $7 \%$, which was hypothesized to be due to the large bore cystoscope used.

Napoléon et al. ${ }^{20}$ described a superficial vascular network (SVN) pattern (Fig. 3) as the diagnostic criterion for serous cystadenoma (SCA). Small and regular structures were seen circulating inside opacified channels that were determined to be vascular structures. This was confirmed upon histological assessment of the surgical specimens. This criterion had an accuracy of $87 \%$, sensitivity of $69 \%$, specificity of $100 \%$, positive predictive value of $100 \%$, and negative predictive value of $82 \%$. The interobserver agreement (IOA) was high $(\kappa=0.77)$. The complication rate in this study was remarkably lower, with only one patient (3\%) developing mild acute pancreatitis.

Subsequently, Napoleon et al. conducted another study (CONTACT 1$)^{21}$ to determine new nCLE criteria for the diagnosis of PCL and perform external retrospective validation. Using this new nCLE criteria, nCLE alone was able to obtain a conclusive diagnosis in 23 of 31 patients (74\%). The accuracy of nCLE criteria was $94 \%$ for mucinous cysts, $87 \%$ for SCA, and $87 \%$ for pseudocysts. A prospective study by Chin et al. ${ }^{22}$ found similar sensitivity (83.3\%) and specificity (75\%) rates for nCLE versus a final histological diagnosis. The overall ac- curacy of nCLE was $80 \%$.

In the CONTACT 2 study, ${ }^{23}$ the larger multicenter prospective study overcame the limitations of a small sample size and the shortage of definitive pathological diagnosis in previous studies. Of the 209 enrolled patients with a noncommunicating solitary cyst, 78 patients with a final diagnosis were included for analysis. The overall diagnostic yield of nCLE was 91\%, while the sensitivity and specificity for the main PCL types were higher than $95 \%$. For SCA and premalignant mucinous cysts, nCLE had a specificity of $100 \%$. The area under the curve for nCLE was significantly higher when compared to CEA (for distinguishing mucinous from non-mucinous cysts; $p<0.01$ ) and EUS morphology (for differentiating between premalignant and benign PCLs; $p<0.05$ ). However, nCLE was not adequately specific for the diagnosis of neuroendocrine neoplasms (NENs) and pseudocysts. In the study, the diagnostic criteria for NENs were fulfilled by one cystic solid pseudopapillary neoplasia, one cystic lymphoma, and one pseudocyst. The diagnostic criteria for pseudocyst were fulfilled by one mucinous lesion and one SCA. Although the effect of nCLE on patient management was not specifically assessed in this study, nCLE achieved a conclusive diagnosis for $91 \%$ of patients who had an inconclusive EUS-FNA diagnosis, and its diagnosis was $100 \%$ accurate when correlated with histology.

A recent publication on the prospective CONfocal endomicroscopy in CYSTic lesions of the pancreas trial by Keane et $\mathrm{al}^{24}$ showed that EUS-nCLE had an overall sensitivity of $79.6 \%$ for all pancreatic lesions. This ranged from 55.6\% for SCA to

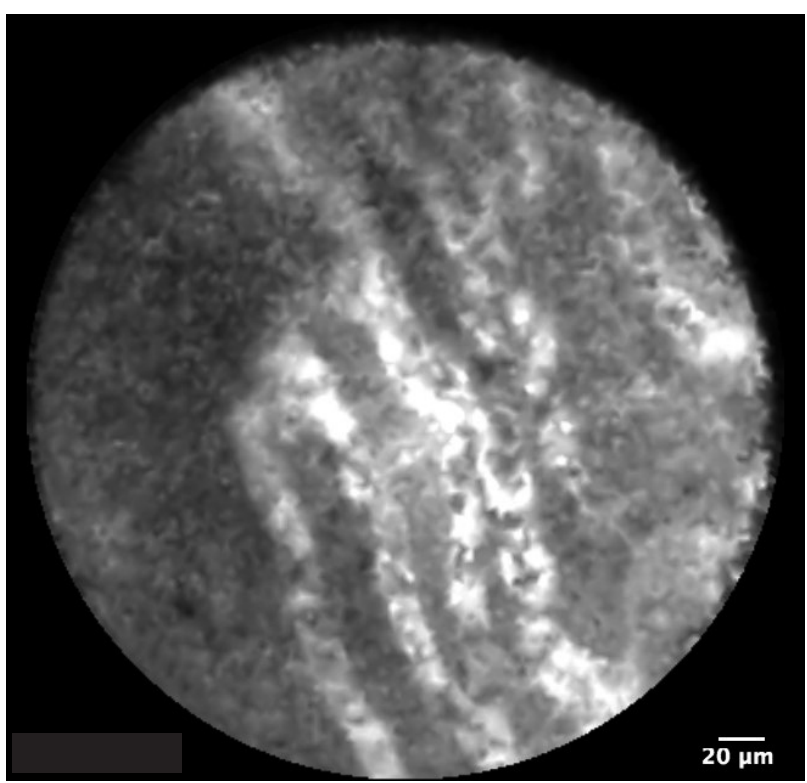

Fig. 3. Superficial vascular network visible on needle-based confocal laser endomicroscopy of serous cystadenoma. 
90\% for IPMN and $100 \%$ for pancreatic ductal adenocarcinoma. The overall accuracy of nCLE was $76.8 \%$. There was a low rate of adverse events (3.5\%) with one case of transient pruritus attributed to a fluorescein allergy and one patient having an infected pseudocyst.

The IOA analyses showed heterogeneous results. In the CONTACT 1 study, ${ }^{21}$ the IOA was assessed of four external reviewers. The diagnostic accuracy for mucinous cysts was 94\%, while the global IOA was substantial ( $\kappa=0.72$; 95\% CI, $0.52-0.87)$. In a study by Karia et al., ${ }^{25} 15$ de-identified nCLE video clips of PCLs were sent to six interventional endoscopists at five institutions. The mean accuracy of these observers was $46 \%$ (range, 20\%-67\%). The low accuracy rates were postulated to be due to poor image quality and the presence of a steep learning curve that the study endoscopists had yet to surmount.

A study by Kadayifci et al. ${ }^{26}$ showed a sensitivity, specificity, and diagnostic accuracy of the findings of epithelial structures by nCLE of $66 \%, 100 \%$, and $80 \%$, respectively, for a mucinous cyst diagnosis. In a study by Krishna et al., ${ }^{27}$ nCLE was performed in 49 participants; of them, a definitive diagnosis was obtained in 26 patients (53.1\%). The overall sensitivity, specificity, and accuracy for diagnosing mucinous PCL were $94 \%, 82 \%$, and $89 \%$, respectively. Substantial IOA and intraobserver reliability (IOR) were achieved for the detection of all nCLE criteria and differentiation of mucinous from non-mucinous cysts $(\kappa=0.67 ; 95 \% \mathrm{CI}, 0.57-0.77$; and $\kappa=0.78 \pm 0.13$, respectively). In another study of Krishna et al., ${ }^{28}$ six endosonographers (each of whom had performed nCLE > 30 times) reviewed the nCLE images of PCLs from 29 participants with surgical $(n=23)$ or clinical $(n=6)$ correlations while blinded to the other clinical data. The overall sensitivity, specificity, and accuracy for the diagnosis of mucinous PCL were 95\%, $94 \%$, and 95\%, respectively. The obtained IOA and IOR were almost perfect $(\kappa=0.81 ; 95 \% \mathrm{CI}, 0.71-0.90$; and $\kappa=0.86 \pm 0.11$, respectively). Importantly, nCLE was $98 \%$ accurate at diagnosing SCA and the IOA and IOR for recognizing the fern pattern (previously known as SVN) were also almost perfect $(\kappa=0.83$; 95\% CI, 0.73-0.92; and $\kappa=0.85 \pm 0.11$, respectively). The same group performed a post hoc analysis ${ }^{29}$ of the EUS-nCLE characteristics of IPMNs. Increased papillary epithelial "width" and "darkness" were the most sensitive (90\% [95\% CI, 84\%-94\%] and $91 \%$ [95\% CI, 85\%-95\%], respectively) and accurate (85\% [95\% CI, 78\%-90\%] and 84\% [95\% CI, 77\%-89\%], respectively) variables with substantial $(\kappa=0.61$ [95\% CI, 0.51-0.71]) and moderate $(\kappa=0.55$ [95\% CI, $0.45-0.65]$ ) IOAs for detecting high-grade dysplasia/adenocarcinoma (HGD-Ca) in cases of IPMN. A papillary width $\geq 50 \mu \mathrm{m}$ and papillary darkness $\leq 90$ pixel intensity had receiver operating characteristic curves of 0.95 and 0.90 , respectively. These quantifications identified HGD-Ca in cases of IPMN with high accuracy.

Palazzo et al. ${ }^{30}$ reported similar findings of a retrospective analysis of 209 cases. The addition of nCLE to EUS-FNA led to substantial changes in management for $28 \%$ of patients in the study $(p<0.001)$. In fact, nCLE increased the IOA from 0.36 (95\% CI, 0.33-0.49) to 0.64 (95\% CI, 0.61-0.67) $(p<0.0001)$ and improved the rates of full agreement among the five experts from $30 \%$ to $54 \%(p<0.0001)$. With nCLE, the surveillance rate of benign SCAs decreased from 40\% (28/70) to $5 \%(4 / 76)$. The addition of nCLE to EUS-FNA significantly improved the reliability of the PCL diagnosis and could impact overall management strategies.

\section{DISCUSSION}

PCLs are commonly detected. Distinguishing benign lesions from those requiring intervention remains difficult, while the adequate management of pancreatic cysts is challenging. After nCLE was shown to be technically feasible in 2011, multiple prospective studies described the appearance of various PCNs under nCLE and correlated them against the histological findings. This was followed by larger multicenter studies that showed the improved diagnostic accuracy of nCLE, which is also useful for obtaining a diagnosis in patients with a previously inconclusive EUS-FNA result with an accuracy comparable to that of surgical histopathology. The presence of a SVN

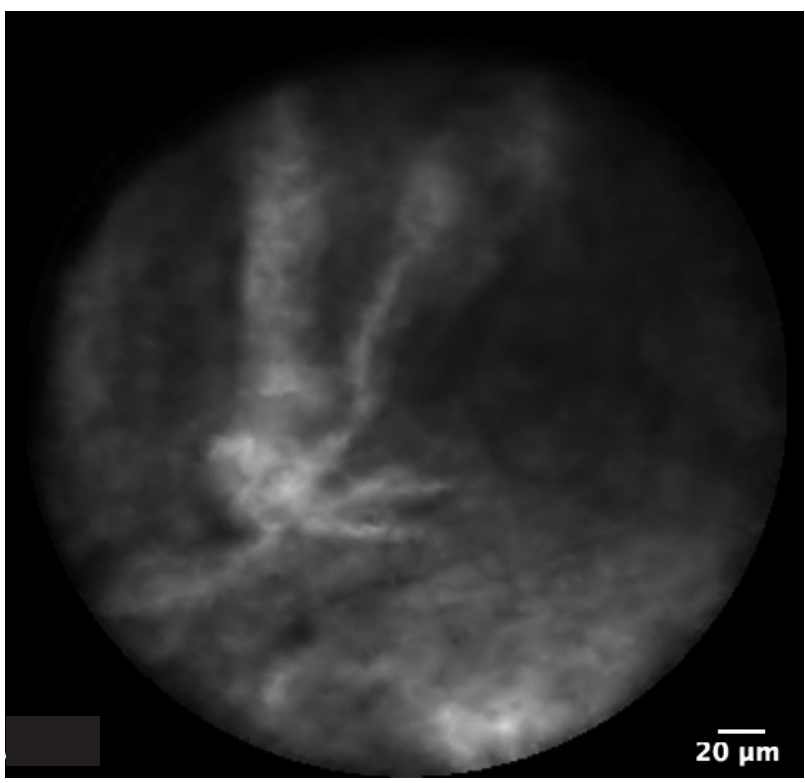

Fig. 4. Papillary projections characterized by a vascular core (white) surrounded by an epithelial border (gray) are visible on needle-based confocal laser endomicroscopy of intraductal papillary mucinous neoplasm. 


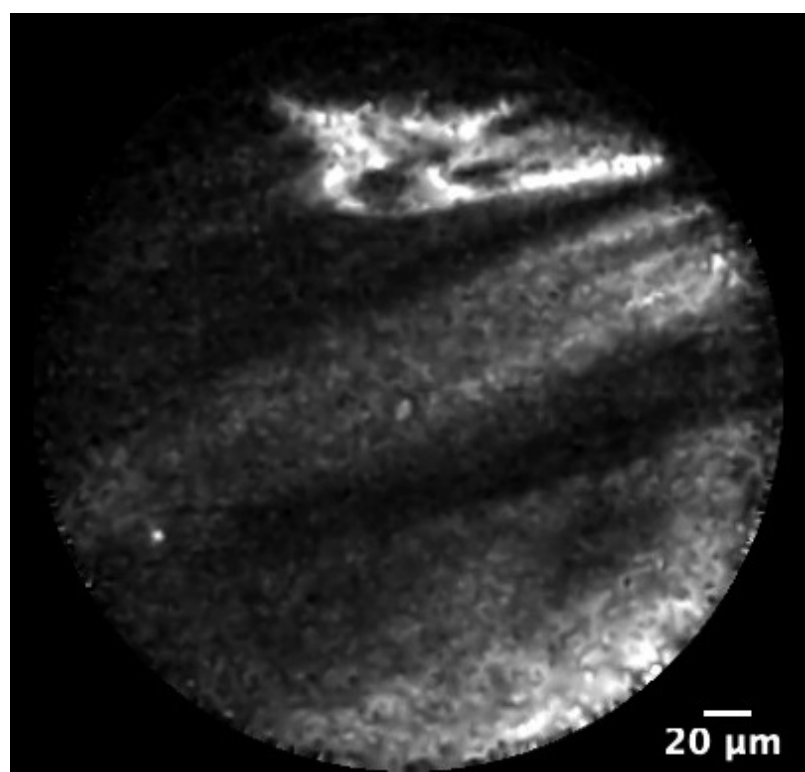

Fig. 5. A thick epithelial band is visible on needle-based confocal laser endomicroscopy of mucinous cystadenoma.

is exclusive to SCA. However, the sensitivity of SVN is limited by epithelium denudation. Oligocystic SCA may not display this characteristic vascular pattern. ${ }^{31}$ The presence of papillary projections or thick epithelial bands is highly specific for mucinous lesions like IPMN (Fig. 4) and MCA (Fig. 5), respectively. The lack of uniform distribution of the epithelium of IPMN and the presence of inflammatory changes may result in the diagnostic features remaining undetected. Pseudocysts (Fig. 6) have a fibrous wall that is paucicellular and avascular and appears as a dark background. Bright uniform particles in clusters contrast prominently against the dark background. These bright particles are likely to be macrophages, which are known to be autofluorescent.

Existing studies have reported several complications related to nCLE (Table 4). The most common complication is acute pancreatitis, with an incidence of $1.3 \%-12 \%$. Few factors have been identified as associated with higher complication of acute pancreatitis-namely the use of a Spyglass cholangioscope (Boston Scientific, Natick, MA, USA) for cystoscopy and post-loading the nCLE probe into the needle. Self-limiting intracystic bleeding was reported from one series of three patients. A prolonged procedure duration and the brushing of the cyst lining with a needle tip were identified as risk factors for such complications. ${ }^{18}$ Various maneuvers improved the safety of the EUS-nCLE examination, such as preloading the nCLE probe before the start of the procedure, avoiding interposition of the pancreatic duct and blood vessels during the FNA, refraining from rubbing the tip of the probe against

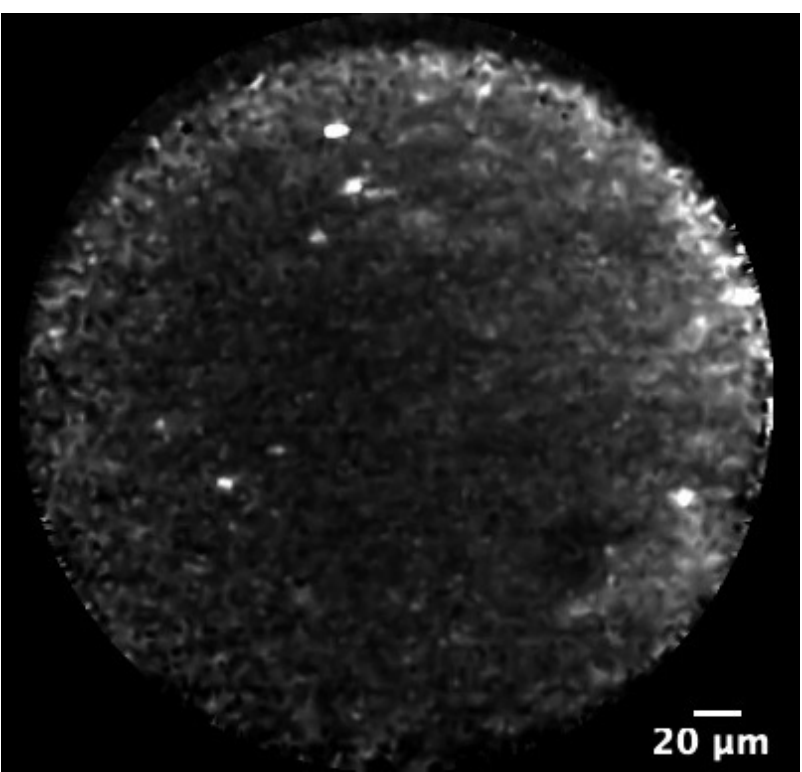

Fig. 6. Bright uniform particles are visible against a dark background on needle-based confocal laser endomicroscopy of pseudocyst.

the cyst wall during the examination, and limiting the nCLE procedure duration to 10 minutes or less. To date, there are no reported cases of fatalities attributed to nCLE.

The use of nCLE is limited by the presence of a significant learning curve. The surmounting of this learning curve requires not only understanding the technical aspect of setting up the device but also the ability to interpret the image sequences. Krishna et al. ${ }^{28}$ reported that acute pancreatitis occurred almost exclusively in the initial few cases of their series, highlighting the importance of overcoming the learning curve. While the manufacturer offers online learning modules to assist the endosonographer's learning process, further formal training initiatives are required. Another challenge of nCLE is that it can only examine approximately $50 \%$ of the cyst wall lining due to the trajectory of the FNA needle precluding the examination of the cyst wall behind it. Thus, the result may not be completely representative of the pathology of the PCL given that the cyst wall epithelium is known to be heterogenous.

Additionally, nCLE has been shown to improve the cost effectiveness of pancreatic cyst management. Although the use of nCLE incurs a significant upfront cost, a cost effectiveness analysis by Le Pen et al. ${ }^{32}$ found that, when used in conjunction with EUS-FNA, the diagnostic accuracy of nCLE is improved, reducing the number of unnecessary surgical procedures and follow-up appointments. The number of false-negative results was also reduced, leading to reduced morbidity-related costs. ${ }^{33}$ 
Table 4. Complications of Needle-Based Confocal Laser Endomicroscopy for Pancreatic Cystic Lesions

\begin{tabular}{|c|c|c|c|c|c|c|c|}
\hline \multirow{2}{*}{ Study } & \multirow{2}{*}{$n$} & \multicolumn{6}{|c|}{ Complications } \\
\hline & & Pancreatitis & Bleeding & Infection & Fluorescein- related & Death & Overall \\
\hline Konda et al. $(2011)^{17}$ & 18 & $2(12 \%)$ & 0 & 0 & 0 & 0 & $2(12 \%)$ \\
\hline Konda et al. $(2013)^{18}$ & 66 & $2(3.0 \%)$ & $3(4.5 \%)$ & 0 & 0 & 0 & $6(9.1 \%)$ \\
\hline Nakai et al. $(2015)^{19}$ & 30 & $2(6.7 \%)$ & 0 & 0 & 0 & 0 & $2(6.7 \%)$ \\
\hline Napoléon et al. $(2015)^{20}$ & 31 & $1(3.2 \%)$ & $4(12.9 \%)$ & 0 & 0 & 0 & $5(16.1 \%)$ \\
\hline Krishna et al. $(2016)^{27}$ & 49 & $3(6.1 \%)$ & 0 & 0 & 0 & 0 & $3(6.1 \%)$ \\
\hline Chin et al. $(2018)^{22}$ & 12 & 0 & $1(8.3 \%)$ & 0 & 0 & 0 & $1(8.3 \%)$ \\
\hline Napoleon et al. $(2019)^{23}$ & 78 & $1(1.3 \%)$ & $1(1.3 \%)$ & 0 & 0 & 0 & $2(2.6 \%)$ \\
\hline Keane et al. $(2019)^{24}$ & 56 & 0 & 0 & $1(1.8 \%)$ & $1(1.8 \%)$ & 0 & $2(3.6 \%)$ \\
\hline Palazzo et al. $(2019)^{30}$ & 209 & $3(1.4 \%)$ & 0 & 0 & 0 & 0 & $3(1.4 \%)$ \\
\hline Overall & 549 & $14(2.6 \%)$ & $9(1.6 \%)$ & $1(0.2 \%)$ & $1(0.2 \%)$ & $0(0.0 \%)$ & $27(4.9 \%)$ \\
\hline
\end{tabular}

\section{CONCLUSIONS}

PCL is increasingly detected due to the widespread use of advanced imaging technique. However, the challenge remains regarding how to ascertain the exact nature of the PCL and select high-risk lesions for surgical resection while preventing oversurveillance or unnecessary procedures in patients with benign lesions. Here we highlighted the increasing positive evidence for nCLE in the assessment of PCL since it provides valuable information in the diagnostic process for PCLs and is a useful supplement to EUS-FNA. The limitations of its high upfront operating cost and steep learning curve must be addressed before nCLE can be widely used and incorporated into future guidelines as a standard of care for the assessment of PCL.

Conflicts of Interest

Damien Meng Yew Tan is a consultant for Boston Scientific. The other authors have no potential conflicts of interest.

Funding

None.

\section{Author Contributions}

Conceptualization: Yung Ka Chin, Clement Chun Ho Wu and Damien Meng Yew Tan

Data curation: YKC, CCHW, DMYT

Formal analysis: YKC, CCHW, DMYT

Project administration: YKC, CCHW, DMYT

Supervision: DMYT

Writing-review\&editing: YKC, CCHW, DMYT
ORCID

\section{Yung Ka Chin:}

Clement Chun Ho Wu:

Damien Meng Yew Tan: https://orcid.org/0000-0002-9869-1334 https://orcid.org/0000-0002-7282-0900 https://orcid.org/0000-0002-0816-9375

\section{REFERENCES}

1. Laffan TA, Horton KM, Klein AP, et al. Prevalence of unsuspected pancreatic cysts on MDCT. AJR Am J Roentgenol 2008;191:802-807.

2. de Jong K, Bruno MJ, Fockens P. Epidemiology, diagnosis, and management of cystic lesions of the pancreas. Gastroenterol Res Pract 2012;2012:147465

3. de Jong K, Nio CY, Hermans JJ, et al. High prevalence of pancreatic cysts detected by screening magnetic resonance imaging examinations. Clin Gastroenterol Hepatol 2010;8:806-811.

4. Nougaret S, Mannelli L, Pierredon MA, Schembri V, Guiu B. Cystic pancreatic lesions: from increased diagnosis rate to new dilemmas. Diagn Interv Imaging 2016;97:1275-1285.

5. Tanaka M, Chari S, Adsay V, et al. International consensus guidelines for management of intraductal papillary mucinous neoplasms and mucinous cystic neoplasms of the pancreas. Pancreatology 2006;6:17-32.

6. Khalid A, Brugge W. ACG practice guidelines for the diagnosis and management of neoplastic pancreatic cysts. Am J Gastroenterol 2007; 102:2339-2349.

7. Goh BK, Tan YM, Cheow PC, et al. Cystic lesions of the pancreas: an appraisal of an aggressive resectional policy adopted at a single institution during 15 years. Am J Surg 2006;192:148-154.

8. Karim SAM, Abdulla KS, Abdulkarim QH, Rahim FH. The outcomes and complications of pancreaticoduodenectomy (Whipple procedure): cross sectional study. Int J Surg 2018;52:383-387.

9. Wang J, Ma R, Churilov L, et al. The cost of perioperative complications following pancreaticoduodenectomy: a systematic review. Pancreatology 2018;18:208-220.

10. Elta GH, Enestvedt BK, Sauer BG, Lennon AM. ACG clinical guideline: diagnosis and management of pancreatic cysts. Am J Gastroenterol 2018;113:464-479. 
11. Tanaka M, Fernández-Del Castillo C, Kamisawa T, et al. Revisions of international consensus Fukuoka guidelines for the management of IPMN of the pancreas. Pancreatology 2017;17:738-753.

12. European Study Group on Cystic Tumours of the Pancreas. European evidence-based guidelines on pancreatic cystic neoplasms. Gut 2018;67:789-804.

13. Hutchins GF, Draganov PV. Cystic neoplasms of the pancreas: a diagnostic challenge. World J Gastroenterol 2009;15:48-54.

14. Lee KH, Lee SJ, Lee JK, et al. Prediction of malignancy with endoscopic ultrasonography in patients with branch duct-type intraductal papillary mucinous neoplasm. Pancreas 2014;43:1306-1311.

15. Brugge WR, Lewandrowski K, Lee-Lewandrowski E, et al. Diagnosis of pancreatic cystic neoplasms: a report of the cooperative pancreatic cyst study. Gastroenterology 2004;126:1330-1336.

16. Meining A. Confocal endomicroscopy. Gastrointest Endosc Clin N Am 2009;19:629-635.

17. Konda VJ, Aslanian HR, Wallace MB, Siddiqui UD, Hart J, Waxman I. First assessment of needle-based confocal laser endomicroscopy during EUS-FNA procedures of the pancreas (with videos). Gastrointest Endosc 2011;74:1049-1060.

18. Konda VJ, Meining A, Jamil LH, et al. A pilot study of in vivo identification of pancreatic cystic neoplasms with needle-based confocal laser endomicroscopy under endosonographic guidance. Endoscopy 2013;45:1006-1013.

19. Nakai Y, Iwashita T, Park DH, Samarasena JB, Lee JG, Chang KJ. Diagnosis of pancreatic cysts: EUS-guided, through-the-needle confocal laser-induced endomicroscopy and cystoscopy trial: DETECT study. Gastrointest Endosc 2015;81:1204-1214.

20. Napoléon B, Lemaistre AI, Pujol B, et al. A novel approach to the diagnosis of pancreatic serous cystadenoma: needle-based confocal laser endomicroscopy. Endoscopy 2015;47:26-32.

21. Napoleon B, Lemaistre AI, Pujol B, et al. In vivo characterization of pancreatic cystic lesions by needle-based confocal laser endomicroscopy (nCLE): proposition of a comprehensive nCLE classification confirmed by an external retrospective evaluation. Surg Endosc 2016;30:2603-2612.

22. Chin YK, Khor CJL, Goh BKP, Lim TKH, Tan DMY. The clinical evaluation of needle-based confocal laser endomicroscopy in the assessment of pancreatic cystic lesion: a pilot study. Proceedings of Singapore
Healthcare 2018;27:96-102.

23. Napoleon B, Palazzo M, Lemaistre AI, et al. Needle-based confocal laser endomicroscopy of pancreatic cystic lesions: a prospective multicenter validation study in patients with definite diagnosis. Endoscopy 2019;51:825-835.

24. Keane MG, Wehnert N, Perez-Machado M, et al. A prospective trial of CONfocal endomicroscopy in CYSTic lesions of the pancreas: CONCYST-01. Endosc Int Open 2019;7:E1117-E1122.

25. Karia K, Waxman I, Konda VJ, et al. Needle-based confocal endomicroscopy for pancreatic cysts: the current agreement in interpretation. Gastrointest Endosc 2016;83:924-927.

26. Kadayifci A, Atar M, Basar O, Forcione DG, Brugge WR. Needle-based confocal laser endomicroscopy for evaluation of cystic neoplasms of the pancreas. Dig Dis Sci 2017;62:1346-1353.

27. Krishna SG, Swanson B, Hart PA, et al. Validation of diagnostic characteristics of needle based confocal laser endomicroscopy in differentiation of pancreatic cystic lesions. Endosc Int Open 2016;4:E1124-E1135.

28. Krishna SG, Brugge WR, Dewitt JM, et al. Needle-based confocal laser endomicroscopy for the diagnosis of pancreatic cystic lesions: an international external interobserver and intraobserver study (with videos). Gastrointest Endosc 2017;86:644-654.e2.

29. Krishna SG, Hart PA, DeWitt JM, et al. EUS-guided confocal laser endomicroscopy: prediction of dysplasia in intraductal papillary mucinous neoplasms (with video). Gastrointest Endosc 2020;91:551-563.e5.

30. Palazzo M, Sauvanet A, Gincul R, et al. Impact of needle-based confocal laser endomicroscopy on the therapeutic management of single pancreatic cystic lesions. Surg Endosc 2019 Aug 13 [Epub]. https://doi. org/10.1007/s00464-019-07062-9.

31. Alvarez-Sánchez MV, Napoléon B. New horizons in the endoscopic ultrasonography-based diagnosis of pancreatic cystic lesions. World J Gastroenterol 2018;24:2853-2866.

32. Le Pen C, Palazzo L, Napoléon B. A health economic evaluation of needle-based confocal laser endomicroscopy for the diagnosis of pancreatic cysts. Endosc Int Open 2017;5:E987-E995.

33. Krishna SG, Swanson B, Conwell DL, Muscarella P 2nd. In vivo and ex vivo needle-based confocal endomicroscopy of intraductal papillary mucinous neoplasm of the pancreas. Gastrointest Endosc 2015;82:571572 . 